\title{
Action planning as predictor of health protective and health risk behavior: an investigation of fruit and snack consumption Liesbeth van Osch ${ }^{1}$, Mariëlle Beenackers ${ }^{1}$, Astrid Reubsaet ${ }^{1}$, Lilian Lechner*2, Math Candel ${ }^{3}$ and Hein de Vries ${ }^{1}$
}

Address: ${ }^{1}$ Department of Health Education and Health Promotion, Maastricht University, Care and Public Health Research Institute (CAPHRI), PO Box 616, 6200 MD, Maastricht, the Netherlands, ${ }^{2}$ Faculty of Psychology, Open University Netherlands, Care and Public Health Research Institute (CAPHRI), PO Box 2960, 6401 DL, Heerlen, the Netherlands and ${ }^{3}$ Department of Methodology and Statistics, Maastricht University, Care and Public Health Research Institute (CAPHRI), PO Box 616, 6200 MD, Maastricht, the Netherlands

Email: Liesbeth van Osch - Liesbeth.vanOsch@gvo.unimaas.nl; Mariëlle Beenackers - M.Beenackers@alumni.unimaas.nl; Astrid Reubsaet - A.Reubsaet@gvo.unimaas.nl; Lilian Lechner* - Lilian.Lechner@ou.nl; Math Candel - Math.Candel@STAT.unimaas.nl; Hein de Vries - Hein.deVries@gvo.unimaas.nl

* Corresponding author

Published: 13 October 2009

International Journal of Behavioral Nutrition and Physical Activity 2009, 6:69 doi:10.1 I86/1479-5868-6-69

This article is available from: http://www.ijbnpa.org/content/6/1/69

(C) 2009 van Osch et al; licensee BioMed Central Ltd.

This is an Open Access article distributed under the terms of the Creative Commons Attribution License (http://creativecommons.org/licenses/by/2.0), which permits unrestricted use, distribution, and reproduction in any medium, provided the original work is properly cited.

\begin{abstract}
Background: Large discrepancies between people's intention to eat a healthy diet and actual dietary behavior indicate that motivation is not a sufficient instigator for healthy behavior. Research efforts to decrease this 'intention - behavior gap' have centered on aspects of self-regulation, most importantly selfregulatory planning. Most studies on the impact of self-regulatory planning in health and dietary behavior focus on the promotion of health protective behaviors. This study investigates and compares the predictive value of action planning in health protective behavior and the restriction of health risk behavior.

Methods: Two longitudinal observational studies were performed simultaneously, one focusing on fruit consumption ( $N=572)$ and one on high-caloric snack consumption $(N=585)$ in Dutch adults. Structural equation modeling was used to investigate and compare the predictive value of action planning in both behaviors, correcting for demographics and the influence of motivational factors and past behavior. The nature of the influence of action planning was investigated by testing mediating and moderating effects.

Results: Action planning was a significant predictor of fruit consumption and restricted snack consumption beyond the influence of motivational factors and past behavior. The strength of the predictive value of action planning did not differ between the two behaviors. Evidence for mediation of the intention - behavior relationship was found for both behaviors. Positive moderating effects of action planning were demonstrated for fruit consumption, indicating that individuals who report high levels of action planning are significantly more likely to translate their intentions into actual behavior.

Conclusion: The results indicate that the planning of specific preparatory actions predicts the performance of healthy dietary behavior and support the application of self-regulatory planning in both health protective and health risk behaviors. Future interventions in dietary modification may turn these findings to advantage by incorporating one common planning protocol to increase the likelihood that good intentions are translated into healthy dietary behavior.
\end{abstract}




\section{Background}

Achieving and maintaining a healthy diet is all about consuming adequate amounts of wholesome nutrition and restricting the consumption of unhealthy, high-caloric foods. If only motivation would be enough for people to eat healthily, we would not be faced with the alarming figures on overweight and obesity. In the Netherlands, high levels of motivation (e.g., 85\% of non-obese adults have a positive intention to prevent weight gain, [1]) sharply contrast with the approximate doubling of obesity prevalence over the last twenty years. Moreover, although approximately 60 to $80 \%$ of the Dutch adult population indicate that they intend to eat more fruit and less fat [24 ], only $30 \%$ of Dutch adults consume sufficient amounts of fruit and approximately one in ten individuals comply with recommendations on saturated fat intake [5].

This discrepancy between motivation and actual health behavior has been subject to profound scrutiny in the past decade. In a review of health behaviors [6], it was demonstrated that only $53 \%$ of individuals with positive intentions to engage in a health behavior translate their intentions into actual behavior. Furthermore, intentions have generally been found to account for only $20 \%$ to $40 \%$ of variance in behavior and behavior change [7-9]. These findings conflict with most traditional social-cognitive theories, such as the Theory of Planned Behavior [10], Social Cognitive Theory [11], and Protection Motivation Theory [12], that consider intention as the most proximal and powerful predictor of health behavior and indicate that other, postmotivational processes are essential in the translation of intentions into behavior. Research efforts to narrow the intention - behavior gap have centered on aspects of self-regulation $[13,14]$ and have resulted in the proposition of planning cognitions as an important volitional factor affecting behavior and behavior change. Notions of these efforts are reflected in recent social cognition models such as the Health Action Process Approach [15] and the I-Change Model [16,17], that acknowledge and demonstrate he importance of self-regulatory planning as an important factor in the translation of intentions into behavior.

Action planning encompasses setting goals and planning specific actions in the striving for these goals. A substantial amount of studies that have recently been performed with regard to the impact of action planning have centered on the concept of implementation intentions [18,19] and related planning concepts in which plans are formulated that specify when, where, and how one intends to perform a specific behavior [20,21]. Several published experimental studies have demonstrated efficacy of action planning in the promotion of health behaviors, such as physical activity [e.g., [21,22], but see [23]] and healthy dietary intake [[24-26]; but see [27]; for reviews see [28,29]]. Furthermore, several correlational studies point towards action planning as a potentially important cognition in the transition of intentions to health behaviors [e.g., [3032]]. With regard to the nature of its behavioral influence, action planning has been found to mediate as well as moderate the intention - behavior relationship [e.g., [3235]].

However, contrary to a relatively large amount of studies that examine the influence of action planning with regard to health promoting and health protective behaviors, there is a notable lack of studies investigating its influence on health risk behaviors, i.e. behaviors that should be reduced, ceased or prevented in order to benefit health [28], such as smoking, (excessive) alcohol consumption, and the consumption of unhealthy foods. In these latter 'avoidance behaviors', the goal behavior is to suppress and avoid an unwanted response (e.g. eating an unhealthy snack), whereas so far, most literature with regard to action planning has focused on 'approach behaviors' that imply the initiation of a desired response (e.g. eating fruit). With regard to nutrition behavior, there have been only four studies published that report on the influence of planning on the restriction of unhealthy eating $[24,26,35,36]$. Although these studies varied in the applied forms of planning - the content of the formulated plans ranged from distraction-inhibition [35] to approach goals [24,26] and avoidance goals [36] - they tentatively indicate that action planning may be effectively applied to the restriction of health risk behaviors. The study by Verplanken and Faes, however, demonstrated that although the planning manipulation resulted in healthier dietary behavior, it did not break the negative influence of counterintentional, unhealthy habits, such as eating fatty snacks and sweets.

All but one study [35] used general dietary assessments as their main outcome measure (e.g. mean daily calorie or fat intake), which makes it difficult to unravel the origin of dietary changes and compromises the interpretation of the effects of action planning in restricting unhealthy eating; a reduction in mean caloric intake can be brought about by a decrease in unhealthy, high-caloric food intake, as well as an overall lower food intake of both unhealthy and healthy foods, or even a lower intake of healthy foods. In order to ascertain that a decrease in unhealthy food consumption is the single cause of reductions in general measures of caloric or fat intake, congruence between the content of action plans and the outcome measures is required. Therefore, consumption measures of separate food categories (e.g. healthy snacks vs. unhealthy snacks) are necessary.

Taken together, these findings and considerations establish the need for a more thorough investigation and comparison of the influence of action planning in the promotion of health protective behaviors and the restric- 
tion of health risk behaviors. Outcomes of this comparison may be particularly relevant in the area of dietary behavior change as achieving and maintaining a healthy diet implies both types of behaviors (i.e. the consumption of healthy foods should be increased, whereas the consumption of unhealthy foods should be decreased). If action planning proves to be important in bridging the intention - behavior gap of both types of behaviors, future interventions may benefit from the use of a single type of planning and one common planning protocol for dietary behavior change.

In line with this reasoning, the present manuscript describes two separate, yet simultaneously performed, longitudinal studies that investigate and compare the predictive value of action planning in health protective behavior and health risk behavior with regard to nutrition. The health protective behavior under study is the consumption of fruit; the health risk behavior pertains to the restricted consumption of high-caloric snacks. Where most previous observational studies have failed to incorporate a measure of past behavior, which is generally the most powerful predictor of future behavior, the present study adequately accounted for the influence of past behavior. This enabled the investigation of the value of action planning in the prediction of behavior as well as behavior change. We hypothesized that action planning would positively predict the performance of both health protective behavior and the restriction of the health risk behavior (hypothesis 1). Based on previous findings with regard to the nature of the influence of action planning [e.g., [30-34,37]], we furthermore hypothesized that action planning mediates as well as moderates the intention - behavior relationship (hypothesis 2). Lastly, we expected that the predictive value of action planning would be equally strong in both types of behaviors (hypothesis 3).

The action planning concept under investigation involves the planning of specific (preparatory) actions [e.g., [34,38-41]] that facilitate the performance of the ultimate goal behavior. This type of action planning is based on goal-setting theory $[42,43]$, assuming that when people are faced with specific goals (e.g. daily consumption of fruit), they tend to formulate plans and task strategies on how the goal can be reached $[42,44,45]$. The development of these action plans predetermines a consecutive course of action (e.g., buying fruit, taking fruit along when you go to work, substituting snacks by fruit, etcetera) that is aimed at facilitating goal achievement.

\section{Methods}

\section{Procedure}

Two separate studies were performed simultaneously, one focusing on fruit consumption and the other focusing on the consumption of high-caloric snacks. Both study sam- ples consisted of Dutch adults ( $>18$ years) that were all registered members of an online survey panel of a private research company. A total of 806 participants were invited by e-mail to participate in the online study on fruit consumption and 807 participants were invited to participate in the online study on snack consumption. Invitations were study-specific, i.e., it was not possible for individuals to participate in both studies. Participants were explained that confidentiality would be ensured, that the concerning study would comprise three measurements and that they would receive a small incentive (approximately $€ 3$ ) after completing all three questionnaires. By activating a link in the e-mail, participants were directed to the web page where they could fill out the questionnaire.

At the baseline measurement (T1), 572 respondents $(71.0 \%)$ filled out the questionnaire on fruit consumption and 585 respondents $(72.5 \%)$ filled out the questionnaire on snack consumption. In the first follow-up measurement one month later (T2), 498 respondents participated in the fruit study ( $87.1 \%$ of baseline) and 508 respondents participated in the snack study $(86.8 \%$ of baseline), whereas a total of 434 respondents in the fruit study (75.9\% of baseline) and 442 respondents in the snack study (75.6\% of baseline) had completed all three questionnaires at the second follow-up measurement two months after baseline (T3).

\section{Questionnaires}

In the baseline questionnaires of both studies, relevant demographic variables, past behavior (previous fruit or snack consumption) self-efficacy and intention were measured. At T2 (one month after baseline), action planning was measured, and at T3 (two months after baseline) the outcome behavior (current fruit or snack consumption) was assessed. The target behaviors that were mentioned in all questions were 'eating a sufficient amount of fruit each day', which was previously explained to participants as 'two pieces of fruit each day', and 'eating as little high-caloric snacks as possible', i.e. restricting the consumption of snacks.

\section{Demographics (TI)}

Gender, age, and highest completed educational level were inquired after. Educational level was categorized into 'low' (elementary education, medium general secondary education, preparatory vocational school, or lower vocational school), 'medium' (higher general secondary education, preparatory academic education, or medium vocational school) and 'high' (higher vocational school or university level).

\section{Self-efficacy $(T I)$}

Self-efficacy expectations were measured by four items in each study and asked to what extent respondents think they will be able to perform the target behavior in various 
situations [e.g., [46]]. For fruit consumption, these situations pertained to 'during the week', 'during the weekend', 'when you are very busy', and 'during the winter months' (Cronbach's $\alpha=0.91$ ). For snack consumption, these situations pertained to 'during the weekend', 'when you are very busy', 'when you are at a party', and 'when you have a craving for snacks' (Cronbach's $\alpha=0.81$ ). Answering options for each item ranged from 'I will certainly not be able to' (1) to 'I will certainly be able to' (7).

\section{Intention ( $T I)$}

Intention was measured by two items in each study. The first item asked to what extent respondents intended to perform the target behavior [e.g., [47]]. In the second item a time-reference was added, asking respondents to what extent they intended to perform the target behavior in the next month [e.g., $[48,49]]$. For both questions, answering options ranged from 'I definitely do not intend to' (1) to 'I definitely intend to' (7). Reliability of the intention measure was high in both studies (fruit: Cronbach's $\alpha=$ 0.93; snack: Cronbach's $\alpha=0.96$ ).

\section{Action planning (T2)}

Action planning was assessed by five items in each study. Items were derived from literature review [e.g., [50-52]] and expert consulting, and assessment was based on techniques used by van Osch and colleagues [34] and de Vries and colleagues [17]. Respondents were asked to what extent they planned to perform several actions or preparatory behaviors in order to reach the target behavior.

With regard to fruit consumption, specific actions that followed the item stem 'Do you have a plan to...' pertained to 'buy (more) fruit?', 'eat fruit at a fixed time of day?', 'put a fruit basket on the table?', 'take fruit along with you when you go somewhere?', and 'replace unhealthy snacks by fruit?' (Cronbach's $\alpha=0.75$ ).

For snack consumption, action planning used the same item stem 'Do you have a plan to...', and specific actions pertained to 'buy less snacks?', 'buy healthy alternatives for snacks?', 'refrain from eating snacks at a fixed time of day?', 'substitute snacks by healthy alternatives?', and 'take healthy alternatives along with you when you go somewhere?' (Cronbach's $\alpha=0.92)$. Answering options for all items ranged from 'I definitely do not' (1) to 'I definitely do' (7).

\section{Fruit consumption $(T I, T 3)$}

The measurement of fruit consumption was based on a validated questionnaire [53] and comprised two items, referring to a) the amount of days in a week the respondent usually eats fruit ( 0 to 7 ), and b) the amount of fruit the respondent averagely consumes on each of these days. Multiplying the responses to these two questions gives a proper overview of the amount of fruit consumed during a week (Spearman correlation coefficients with two 24hour consumption recalls $=0.68$ for men, 0.75 for women; correct tertile classification $=52 \%$ ] [53] .

Snack consumption (TI, T3)

The measurement of snack consumption was based on previous questionnaires $[49,54,55]$ and consisted of five items, measuring the consumption of five types of highcaloric snacks: 1. fatty snacks (e.g. hamburgers, pizza), 2. salty snacks (e.g. nuts, potato chips), 3 . sugary snacks (e.g. cake, cookies), 4. candy bars, and 5. savory snacks (e.g. dices of cheese, sausage). Respondents were asked to indicate how many times per week they consumed each of the forenamed types of snacks. Answering options ranged from 'Never or less than once a week' (1) to 'Every day' (8). The five scores were added to indicate the total amount of snacks consumed per week.

\section{Statistical analysis}

Structural Equation Modeling with Mplus 4.1 (Muthen \& Muthen, 1998-2006), using Maximum Likelihood (ML) estimation was used to test hypothesized associations between the various cognitive constructs. Background variables (age, sex, and educational level) and behavioral measures of fruit and snack consumption were observed variables. Self-efficacy, intention, and action planning were latent constructs, measured by their separate indicators, as defined in the description of the questionnaires. In the basic models, intention and self-efficacy were modeled as direct predictors of behavior, whereas self-efficacy also had an indirect influence through intention. In order to assess the contribution of action planning in the prediction of both behaviors (hypothesis 1), a constrained pathway between action planning and the outcome behavior was included in the basic models. In the extended models, this relationship was freed and estimated. All models were corrected for the background variables. Past behavior was later added to the model and was thought to be correlated with intention and self-efficacy, and directly predictive of current behavior.

The moderation hypothesis (hypothesis 2) was tested using the Maximum Likelihood with robust standard errors and chi-square (MLR) because of the expected nonnormality of the moderation model, as induced by the inclusion of the intention $\times$ action planning interaction term. The moderation models were compared to a constrained moderation model in which the interaction term was constrained to zero. The Satorra-Bentler scaled chisquare difference test was used to assess the statistical significance of the interaction term [56].

In order to test whether the predictive value of action planning was equally powerful for both behaviors 
(hypothesis 3), independent-samples comparison of correlation coefficients was performed [57].

Model fit was assessed using the Comparative Fit Index (CFI), the Tucker-Lewis-Index (TLI), and the Root-MeanSquare Error of Approximation (RMSEA). For a satisfactory model fit, the CFI and the TLI should be high (> 0.90 ), whereas the RMSEA should be low (preferably < 0.08) [58].

\section{Results}

\section{Description of samples}

Somewhat more than half of the respondents in the fruit consumption study were female $(53.3 \%)$. The mean age of this sample was 47.8 years $(S D=16.0)$ and most respondents had a medium level of education (42.5\%). Approximately one quarter of the respondents was of low educational level $(26.3 \%)$ and $31.2 \%$ had a high educational level.

In the snack consumption study, $48.9 \%$ of respondents were female and the mean age was $49.5(\mathrm{SD}=15.4)$. Again, most respondents had a medium level of education $(37.6 \%)$, whereas $31.3 \%$ had a low level of education and $31.1 \%$ was highly educated. The mean intention towards eating sufficient amounts of fruit $($ Mean $=5.20$; $\mathrm{SD}=$ 1.41) was substantially higher than the intention to restrict the consumption of high-caloric snacks $($ Mean $=$ $3.18 ; \mathrm{SD}=1.62$ ).

Chi-square difference tests and independent-samples ttests did not indicate any demographic differences between the two study samples.

\section{Attrition analyses}

Logistic regression analyses demonstrated that in the snack consumption study, drop-outs $(\mathrm{N}=143)$ were somewhat lower educated than respondents who completed all three measurements $(\mathrm{N}=442)(\mathrm{OR}=0.67,95 \%$ $\mathrm{CI}=0.98-1.02, p=0.03)$. No differences were found between drop-outs and completers with regard to age, sex, self-efficacy, intention and snack consumption at baseline, and action planning at the first follow-up. No indications of selective attrition were found in the fruit consumption study.

\section{Measurement models}

Bivariate correlations between cognitions and outcome behaviors are depicted in Table 1. Self-efficacy tended to correlate most strongly with fruit and snack consumption, whereas action planning correlated most strongly with intention.

Confirmatory factor analyses were performed to test the measurement models with regard to both outcome behav- iors. Both models included 11 items, measuring the three latent variables (self-efficacy, intention, and action planning). All factor loadings in both models were significant with values between 0.48 and 0.98 for the fruit consumption model and between 0.42 and 0.96 for the snack consumption model. The fit of both measurement models was satisfactory (fruit consumption: $\mathrm{CFI}=0.98$, TLI $=$ 0.97, RMSEA $=0.06$; snack consumption: $\mathrm{CFI}=0.95$, TLI $=0.93$, RMSEA $=0.09$ ).

\section{Model results: Fruit consumption Basic model}

The basic model with regard to fruit consumption fitted the data well $(\mathrm{CFI}=0.95$, TLI $=0.94$, RMSEA $=0.06)$. Intention $(\beta=0.13 ; \mathrm{B}=0.62 ; p<0.01)$ and self-efficacy $(\beta$ $=0.47 ; \mathrm{B}=2.02 ; p<0.001$ ) were both significant predictors of fruit consumption, with self-efficacy as the strongest predictor. Age was the only significant demographic predictor of fruit consumption $(\beta=0.15 ; \mathrm{B}=0.06$; $p<$ $0.001)$. Together, they explained $36.2 \%$ of the variance in fruit consumption at T3, eight weeks after baseline.

\section{Predictive value and mediating influence of action planning}

(hypothesis I and 2)

To assess the predictive value of action planning with regard to fruit consumption, action planning was modeled as a mediating variable between intention and behavior and between self-efficacy and behavior. The extended model fitted the data well $(\mathrm{CFI}=0.96, \mathrm{TLI}=0.95$, RMSEA $=0.06)$. Action planning significantly predicted fruit consumption $(\beta=0.20 ; B=1.34 ; p<0.001)$. Self-efficacy retained its behavioral impact $(\beta=0.42 ; \mathrm{B}=1.80$; $p<$ 0.001 ), whereas the influence of intention on fruit consumption was rendered non-significant $(\beta=0.05 ; \mathrm{B}=$ $0.24 ; p>0.10)$. This latter result indicates that action planning fully mediated the relationship between intention and behavior. Action planning itself was positively predicted by both intention $(\beta=0.42 ; \mathrm{B}=0.31 ; p<0.001)$ and self-efficacy $(\beta=0.20 ; \mathrm{B}=0.13 ; p<0.001)$. The extended model accounted for $39.3 \%$ of the variance in fruit consumption.

Table I: Pearson correlations between cognitions, past behavior and current outcome behaviors ${ }^{a, b}$

\begin{tabular}{lccccc}
\hline & I & $\mathbf{2}$ & $\mathbf{3}$ & $\mathbf{4}$ & $\mathbf{5}$ \\
\hline I. Self-efficacy & - & 0.58 & 0.40 & 0.63 & 0.57 \\
2. Intention & 0.38 & - & 0.48 & 0.42 & 0.36 \\
3. Action planning & 0.17 & 0.57 & - & 0.31 & 0.33 \\
4. Past fruit/snack consumption & -0.42 & -0.31 & -0.18 & - & 0.76 \\
5. Fruit/snack consumption & -0.36 & -0.29 & -0.22 & 0.60 & -
\end{tabular}

a All correlations between variables in the fruit consumption study are depicted above the diagonal; correlations between variables in the snack consumption study are depicted below the diagonal

${ }^{b}$ All correlations are significant at the 0.001 level (two-tailed) 
To test whether the inclusion of action planning made a significant contribution to the prediction of fruit consumption, a log-likelihood difference chi-square test was performed. This test resulted in a $\chi^{2}$-value of $12.32(\mathrm{df}=$ $1 ; p<0.001)$, which indicates that adding action planning to the model significantly improved the prediction of fruit consumption.

When past behavior was added to the model, action planning $(\beta=0.13 ; \mathrm{B}=0.87 ; p<0.01)$ and self-efficacy $(\beta=$ $0.10 ; \mathrm{B}=0.43 ; p<0.05$ ) remained significant (see Figure $1)$. Intention did not significantly predict behavior $(\beta=-$ $0.03 ; \mathrm{B}=-0.12 ; p>0.10)$. Past behavior was the most powerful predictor of fruit consumption $(\beta=0.64 ; \mathrm{B}=0.60 ; p$ $<0.001$ ) and increased the explained variance of the model to $60.7 \%$.

\section{Moderating effect of action planning (hypothesis 2)}

In order to estimate the potential moderating effect of action planning in the intention - behavior relationship, an intention $\times$ action planning interaction effect was added to the extended model (without past behavior). All other pathways were left unchanged. The interaction effect between action planning and intention was significant $(\mathrm{t}$ $=2.15 ; p<0.05)$, indicating that action planning is more beneficial when intentions are high. We tested whether the inclusion of this interaction effect would result in a better model for explaining the role of action planning in the volitional phase. The Satorra-Bentler scaled chi-square difference test [56] was used to compare the extended model to the extended model with added interaction effect. In this test, the usual normal-theory chi-square statistic is divided by a scaling correction to better approximate chi-square under non-normality, as is the case when estimating an interaction effect. Calculation of the corrected difference in $-2 \log$ likelihood $(\Delta-2 \mathrm{LL}=-5.04 ; \mathrm{df}=$ $1 ; p<0.01)$ indicated that including the action planning $\times$ intention interaction effect significantly improved model fit.

The moderating effect of action planning was also tested in the presence of past behavior. However, when past behavior was added to the model, the action planning $\times$ intention interaction effect was no longer significant $(\mathrm{t}=$ $0.80 ; p>0.10)$.

\section{Model results: Snack consumption \\ Basic model}

The basic model with regard to snack consumption fitted the data well $(\mathrm{CFI}=0.94$, TLI $=0.93$, RMSEA $=0.07)$. Intention $(\beta=-0.19 ; \mathrm{B}=-0.44 ; p<0.001)$ and self-efficacy $(\beta=-0.31 ; \mathrm{B}=-2.11 ; p<0.001)$ were both significant predictors of snack consumption, with self-efficacy as the strongest predictor. The explained variance of snack consumption $(16.8 \%)$ was substantially lower than that of fruit consumption.

\section{Predictive value and mediating influence of action planning (hypothesis I and 2)}

To assess the predictive value of planning with regard to snack consumption, action planning was modeled as a mediating variable between intention and behavior and

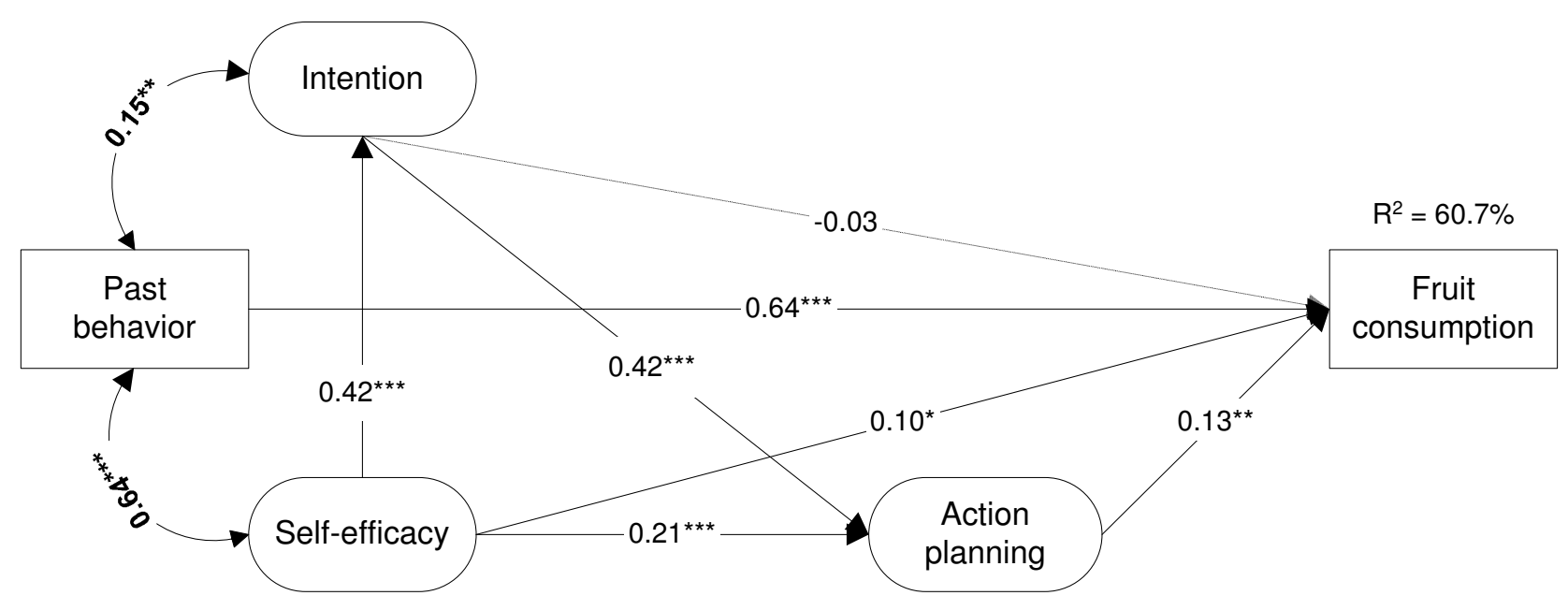

Figure I

Structural equation model with standardized regression coefficients assessing the predictive value of action planning with regard to fruit consumption. $*_{p}<0.05 * *_{p}<0.01 *_{* *}<0.001$ 
between self-efficacy and behavior. This extended model fitted the data rather well $(\mathrm{CFI}=0.95$, TLI $=0.93$, RMSEA $=0.07)$. Action planning was found to be a marginally significant negative predictor of snack consumption $(\beta=-$ $0.10 ; \mathrm{B}=-0.27 ; p=0.06)$, suggesting that higher scores on plans to restrict snack consumption lead to lower snack consumption. Self-efficacy $(\beta=-0.31 ; \mathrm{B}=-2.120 ; p<$ $0.001)$ retained its influence on behavior. Although intention also remained significant $(\beta=-0.13 ; \mathrm{B}=-0.30 ; p<$ $0.05)$, its predictive value was reduced as a result of the inclusion of action planning, indicating partial mediation of action planning in the intention - behavior relationship. Furthermore, intention was a strong predictor of action planning $(\beta=0.57 ; \mathrm{B}=0.51 ; p<0.001)$, whereas the impact of self-efficacy on action planning was practically absent $(\beta=-0.01 ; \mathrm{B}=-0.03 ; p>0.10)$. Together the behavioral determinants explained $17.5 \%$ of the variance in snack consumption.

To test whether the inclusion of action planning made a significant contribution to the prediction of snack consumption, a log-likelihood difference chi-square test was performed. This test resulted in a $\chi^{2}$-value of $3.51(\mathrm{df}=1$; $p=0.06$ ), indicating that adding action planning to the model resulted in a marginally significant improvement of the prediction of snack consumption.

When past behavior was added to the basic model, intention no longer significantly predicted behavior $(\beta=-0.01$;
$\mathrm{B}=-0.03 ; p>0.10$; see Figure 2). Although past behavior was the most powerful predictor of snack consumption $(\beta$ $=0.53 ; \mathrm{B}=0.48 ; p<0.001)$ and increased the explained variance of the model to $38.7 \%$, both action planning $(\beta$ $=-0.11 ; \mathrm{B}=-0.28 ; p<0.05)$ and self-efficacy $(\beta=-0.11 ; \mathrm{B}$ $=-0.73 ; p<0.05)$ were found to be significant predictors of behavior.

\section{Moderating effect of action planning (hypothesis 2)}

An intention $\times$ action planning interaction effect was included in the extended model to test for a potential moderating effect of action planning in the relationship between intention and snack consumption. Past behavior was initially excluded from the analyses; all other pathways were left unchanged.

A small trend towards significance was found for the interaction effect $(\mathrm{t}=-1.56 ; p=0.12)$, which tentatively indicates that action planning may moderate the intention behavior relationship. The Satorra-Bentler scaled chisquare difference test, however, indicated that the corrected difference in -2 log likelihood was non-significant $(\Delta-2 \mathrm{LL}=2.32 ; \mathrm{df}=1 ; p>0.10)$ indicated that including the interaction effect did not improve model fit.

The moderating effect of action planning was also tested in the presence of past behavior. The action planning $\times$ intention interaction effect was not significant in this model $(\mathrm{t}=-0.49 ; p>0.10)$.

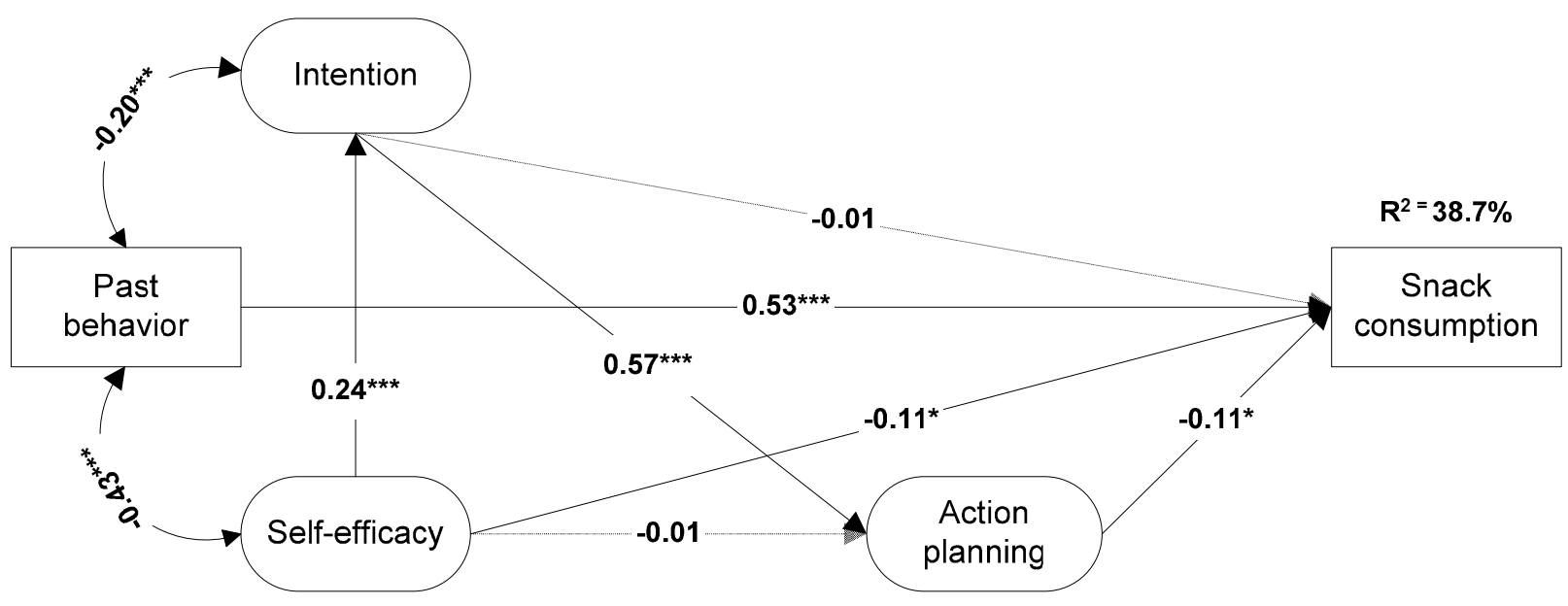

Figure 2

Structural equation model with standardized regression coefficients assessing the predictive value of action planning with regard to snack consumption. $*_{p}<0.05 *^{*} p<0.01 *^{*} * p<0.001$ 
Comparison of predictive value of action planning (hypothesis 3) In order to test whether the predictive value of action planning differed with regard to the two behaviors, independent-samples comparison of correlation coefficients was performed [57]. This test resulted in a $z$-value of 1.50, indicating that the predictive value of action planning did not significantly differ between the two behaviors $(p>$ $0.10)$.

\section{Discussion}

The most important finding of the present study is that action planning significantly predicted health protective behavior (i.e. fruit consumption) as well as the restriction of health risk behavior (i.e., high-caloric snack consumption). Our results showed a better model fit when action plans were added to the model with only attitudes, social influences, self-efficacy and intentions, indicating that the prediction of both types of behavior significantly benefited from the incorporation of action planning, thereby conforming our first hypothesis. When viewed in the light of the literature on other health behaviors, such as physical activity [20-22,31], sun protection behavior [34,59], and (vitamin) pill intake $[60,61]$, our findings with regard to fruit consumption support the notion that action planning may be an important strategy to promote health protective behaviors and suggest that current social-cognitive models on health protective behavior should be extended by incorporating volitional cognitions that facilitate the transition from motivation to behavior. Whereas most previous observational studies that found a behavioral influence of action planning failed to incorporate a measure of past behavior in the analyses, the present study accounted for the influence of past behavior in the extended analyses. Even after the inclusion of past behavior, which is generally the most powerful predictor of future behavior, action planning remained significant, which demonstrates that action planning significantly predicted behavior change. These findings corroborate results from several intervention studies, in which the formation of action plans has been shown to increase the performance of health behaviors [e.g., [20,25]]. The interplay between action planning and past behavior was outside the scope of the present study. Thorough examination of this relationship would, however, be an interesting direction for future research, as this may yield important information on theoretical modeling and practical application of planning strategies in individuals with high and low levels of past behavior.

Our findings with regard to snack consumption verify these suggestions and broaden their scope to include both health protective as well as health risk behaviors. The present study is the first to explicitly compare the predictive value of planning in both types of behaviors and found that the predictive value of action planning was equally powerful in the promotion of fruit consumption and the restriction of snack consumption. These findings confirm our third hypothesis and indicate that one and the same type of planning can be applied in both types of health behaviors.

Other important findings pertain to the established mediating and moderating effects of action planning (hypothesis 2). The longitudinal correlational design of the present study allowed us to examine the nature of the influence that action planning exerts in the intention behavior relationship. Our findings of full mediation in the fruit consumption study and partial mediation in the snack consumption study confirm our hypothesis and correspond to results of previous studies, in which both full [e.g., [32,62,63]] and partial [e.g., [30,33]] mediation have been found in various behaviors. The difference in mediating effects may pertain to the strength of the underlying intentions. Wiedemann and colleagues [64] have demonstrated that the strength of the mediated effect of action planning increases along with levels of intentions. The relatively low intention with regard to restricted snack consumption, as compared to fruit consumption, may therefore have precluded full mediation of the intention behavior relationship by action planning.

The results with regard to potential moderating effects of action planning partially confirm our second hypothesis. A positive moderating effect of action planning was demonstrated in the fruit consumption study, thereby replicating previous reports of moderation of the intention behavior relationship [e.g., [30,33]]. However, only a small trend with regard to the moderation effect was found in the snack consumption study. The insignificance of this effect may, again, be explained by relatively low motivation scores; the overall intention towards restricted snack consumption was substantially lower than the intention to eat sufficient amounts of fruit, which may have precluded the appearance of moderating effects of action planning in the snack consumption study. Besides the proposition to incorporate action planning in existing, traditional social-cognitive models, these findings provide suggestions on how and where to integrate the concept; action planning can tentatively be considered as a mediator as well as moderator in the intention - behavior relationship. It should, however, be mentioned that the present consideration of action planning as concurrent mediator and moderator, is at odds with the conceptualization of moderators as being unaffected by the status of a predictor variable [e.g., [65,66]; but see $[67,68]]$. In the present study, action planning was measured at T2 in order to adequately investigate its mediating influence. Although this measure may be tentatively considered as a proxy for a baseline measure of action planning, application of the latter would have resulted in a 
stricter conceptualization and testing of the moderation effect. This limitation should be taken into account when interpreting the current findings and future studies would do well to incorporate longitudinal measurements of mediating and moderating variables.

Furthermore, whereas the four previous studies used a similar type of action planning [i.e. implementation intentions; $[24,26,35,36]]$, the current study used a different approach. Instead of focusing on when, where, and how a goal-directed response will be implemented (i.e. eating fruit, not eating snacks), the formation of specific preparatory plans was emphasized. Although the former type of planning, i.e. implemental planning, has been subject of substantial research efforts to decrease to intention - behavior gap, the latter planning mode, i.e. preparatory planning, has also been shown to reliably predict a variety of health behaviors [e.g., [17,34,39]]. Moreover, one of our previous studies compared the behavioral influence of both types of behaviors and found that preparatory planning outperformed implemental planning in the prediction of fruit consumption [69]. Further, preferably experimental, research is, however, recommended to substantiate the present findings and optimize planning concepts and interventions for both health protective and health risk behaviors. In doing so, the application of coping planning as a protocol for restriction of health risk behavior may be reckoned with. Coping planning is a barrier-focused strategy that pertains to the identification of risk situations and the specification of suitable coping responses [70]. As this strategy has been shown to reliably predict performance of health behavior in the face of barriers [20,70-72] and has been successfully applied to the restriction of health risk behavior, such as smoking [73] and binge-drinking [74,75], comparison of the benefits of this and other types of planning may yield vital knowledge for the optimization of planning interventions.

Limitations of the present study need to be acknowledged. First, the lack of validity statistics with regard to the behavioral assessment of snack consumption should be mentioned and calls for caution in the interpretation of the results regarding this measure. Items used in the present study were part of a food frequency questionnaire to estimate total and saturated fat intake $[54,55]$ and although this questionnaire has been previously validated, there are currently no specific validity statistics available for the selection of items used to measure snack consumption.

Second, a relatively low explained variance of snacking behavior was found, indicating that other motivational, volitional, and/or environmental factors need to be taken into account for the prediction of snack consumption. The low explained variance is, however, not uncommon, as dietary behavior is generally not well-predicted with explained variances of $30 \%$ and higher being exceptions rather than the rule [76]. Furthermore, although ultimately this study aims at optimizing the prediction of fruit and snack consumption, the primary purpose was to investigate the influence of action planning in the intention - behavior relationship. We therefore only took three other direct predictors of the behaviors into account (past behavior, intention and self-efficacy), whereas most previous studies included many more determinants, often resulting in higher explained variances [e.g., $[49,54,77]]$. Third, data were collected from a random sample of adults that were all members of an existing internet research panel. As these respondents voluntarily participate in surveys and receive incentives for their participation, the degree to which the findings generalize to the Dutch population at large may be limited. However, the demographic characteristics of the participants in both study samples corresponded rather well to demographic distributions within the Dutch adult population [5], rendering substantial reduction of the external validity of our results unlikely. Furthermore, attrition was found to be somewhat selective in the snack consumption sample as lower educated participants were more likely to drop out. This attrition bias may limit internal and external validity of the study. However, general attrition rates were equal in both study samples and the influence of educational level as a covariate was not significant. It is therefore unlikely that the main results of this study have been compromised as a result of attrition.

\section{Conclusion}

Although replication of the findings in preferably experimental settings is required for different behaviors as well as different types of action planning, the present study indicates that action planning may benefit both the actual performance and initiation of healthy behavior and the restriction and suppression of unhealthy behavior. Future interventions in dietary modification may turn these findings to advantage by incorporating one common planning protocol to increase the likelihood that good intentions are translated into healthy dietary behavior.

\section{Competing interests}

The authors declare that they have no competing interests.

\section{Authors' contributions}

$\mathrm{LvO}$ coordinated the conception and design of the study and the acquisition of the data. Furthermore, LvO analyzed and interpreted the data and drafted the manuscript. $\mathrm{MB}$ participated in the data analysis and data interpretation and the drafting of the manuscript. AR and LL participated in the design of the study and the acquisition of the data and critically revised the manuscript. MC substantially participated in the analysis and interpretation of the data and helped to draft the manuscript. HdV was 
involved in the design of the study and the acquisition of the data and critically revised the manuscript. All authors read and approved the final manuscript.

\section{Acknowledgements}

This study was financially supported by a grant of the Dutch Cancer Society (KWF Kankerbestrijding).

\section{References}

I. Wammes B, Kremers S, Breedveld B, Brug J: Correlates of motivation to prevent weight gain: a cross sectional survey. Int $J$ Behav Nutr Phys Act 2005, 2: 1-8.

2. Lechner L, Brug J, de Vries H, van Assema P, Mudde A: Stages of change for fruit, vegetable, and fat intake: consequences of misperception. Health Educ Res 1998, 13: I-II.

3. van Assema P, Ronda G, Steenbakkers M, Quaedvlieg M, Brug J: The reach of a computer-tailored nutrition education program in the Dutch heart health community intervention "Hartslag Limburg". J Nutr Educ Behav 2006, 38:293-297.

4. Verheijden MW, Veen JE van der, van Zadelhoff WM, Bakx C, Koelen MA, Hoogen HJ van der, van Weel C, van Staveren WA: Nutrition guidance in Dutch family practice: behavioral determinants of reduction of fat consumption. Am J Clin Nutr 2003, 77:1058S-1064S.

5. Voedingscentrum: Zo eet Nederland. Resultaten van de voedselconsumptiepeiling 1997-1998 [This is how the Dutch eat: Results of the food consumption survey 1997-1998] Den Haag; 1998.

6. Sheeran P: Intention-behavior relations: a conceptual and empirical review. In European review of social psychology Volume 12. Edited by: Strobe W, Hewstone M. Chichester, UK: Wiley; 2002:I-30.

7. Conner M, Armitage C): Extending the Theory of Planned Behavior: A review and avenues for further research. J Appl Soc Psychol 1998, 28: I429- I 464.

8. Godin G, Kok G: The theory of planned behavior: A review of its applications to health-related behaviors. Am J Health Promot 1995, I I:87-98. (1996)

9. Sutton S: Predicting and explaining intentions and behavior: How well are we doing? J Appl Soc Psychol 1998, 28:1317-1338.

10. Ajzen I: The theory of planned behavior. Organ Behav Hum Decis Process 199I, 50(2): I79-2II.

II. Bandura A: Social foundations of thought and action: A social cognitive theory New York: Prentice Hall; 1986.

12. Rogers RW: Cognitive and physiological processes in fear appeals and attitude change: $A$ revised theory of protection motivation. In Social Psychophysiology - A sourcebook Edited by: Cacioppo JT, Petty RE. New York: Guilford Press; 1983: I53-I76.

13. Carver CS, Scheier MF: On the self-regulation of behavior New York: Cambridge University Press; 1998.

14. Baumeister RF, Heatherton TF, Tice DM: Losing control: How and why people fail at self-regulation San Diego, CA: Academic Press; 1994.

15. Schwarzer R: Self-efficacy in the adoption and maintenance of health behaviors: Theoretical approaches and a new model. In Self-efficacy: Thought control of action Edited by: Schwarzer R. Washington, DC: Hemisphere; 1992:217-242.

16. de Vries H, Mesters I, Steeg $\mathrm{H}$ van de, Honing $\mathrm{C}$ : The general public's information needs and perceptions regarding hereditary cancer: an application of the Integrated Change Model. Patient Educ Couns 2005, 56: 1 54- 165.

17. de Vries H, Mesters I, van 't Riet J, Willems K, Reubsaet A: Motives of Belgian adolescents for using sunscreen: The role of action plans. Cancer Epidemiol Biomarkers Prev 2006, I 5(7): I 360-1366.

18. Gollwitzer PM: Goal achievement: the role of intentions. In European review of social psychology Volume 4. Edited by: Hewstone M, Strobe W. Chichester, UK: Wiley; 1993: I4I-I85.

19. Gollwitzer PM: Implementation intentions: strong effects of simple plans. Am Psychol 1999, 54:493-503.

20. Sniehotta FF, Scholz U, Schwarzer R: Action plans and coping plans for physical exercise: A longitudinal intervention study in cardiac rehabilitation. BrJ Health Psychol 2006, I I:23-37.

21. Ziegelmann JP, Lippke S, Schwarzer R: Adoption and maintenance of physical activity: Planning interventions in young, middleaged, and older adults. Psychol Health 2006, 2 I(2): 145-163.
22. Milne S, Orbell S, Sheeran P: Combining motivational and volitional interventions to promote exercise participation: Protection motivation theory and implementation intentions. Br J Health Psychol 2002, 7(2): I63-I84.

23. De Vet E, Oenema A, Sheeran P, Brug J: Should implementation intentions be implemented in obesity prevention: the impact of if-then plans on daily activity in Dutch adults. Int J Behav Nutr Phys Act 2009, 6: II

24. Armitage $\mathrm{CJ}$ : Evidence that implementation intentions reduce dietary fat intake: A randomized trial. Health Psychol 2004, 23:319-323.

25. Kellar I, Abraham C: Randomized controlled trial of a brief research-based intervention promoting fruit and vegetable consumption. BrJ Health Psychol 2005, 10:543-558.

26. Verplanken B, Faes S: Good intentions, bad habits, and effects of forming implementation intentions on healthy eating. Eur J Soc Psychol 1999, 29:59|-604.

27. Jackson C, Lawton R, Knapp P, Raynor DK, Conner M, Lowe C, Closs $S J$ : Beyond intention: do specific plans increase health behaviours in patients in primary care: $A$ study of fruit and vegetable consumption. Soc Sci Med 2005, 60:2383-2391.

28. Gollwitzer PM, Sheeran P: Implementation Intentions and Goal Achievement: A Meta-Analysis of Effects and Processes. In Advances in Experimental Social Psychology Volume 38. Edited by: Zanna MP. San Diego: Academic Press; 2006:69-119.

29. Sheeran P, Milne S, Webb TL, Gollwitzer PM: Implementation intentions and health behaviour. In Predicting health behaviour Edited by: Conner M, Norman P. Berkshire: Open University Press; 2005:276-323.

30. Jones F, Abraham C, Harris P, Schulz J, Chrispin C: From knowledge to action regulation: Modeling the cognitive prerequisites of sun screen use in Australian and UK samples. Psychol Health 200I, 16(2): 19I-206.

31. Schwarzer R, Schüz B, Ziegelmann JP, Lippke S, Luszczynska A, Scholz $U$ : Adoption and maintenance of four health behaviors: theory-guided longitudinal studies on dental flossing, seat belt use, dietary behavior, and physical activity. Ann Behav Med 2007, 33(2): 156-166.

32. Sniehotta FF, Scholz U, Schwarzer R: Bridging the intentionbehaviour gap: Planning, self-efficacy, and action control in the adoption and maintenance of physical exercise. Psychol Health 2005, 20(2): I 43-160.

33. Norman P, Conner M: The Theory of Planned Behavior and exercise: Evidence for the mediating and moderating roles of planning on intention-behavior relationships. J Sport Exerc Psychol 2005, 27(4):488-504.

34. van Osch L, Reubsaet A, Lechner L, Candel M, Mercken L, de Vries $\mathrm{H}$ : Predicting parental sunscreen use: disentangling the role of action planning in the intention - behavior relationship. Psychol Health 2008, 23:829-847.

35. Achtziger A, Gollwitzer PM, Sheeran P: Implementation intentions and shielding goal striving from unwanted thoughts and feelings. Pers Soc Psychol Bull 2008, 34:38I-393.

36. Sullivan HW, Rothman AJ: When planning is needed: Implementation intentions and attainment of approach versus avoidance health goals. Health Psychol 2008, 27:438-444.

37. Sheeran P, Webb TL, Gollwitzer PM: The interplay between goal intentions and implementation intentions. Pers Soc Psychol Bull 2005, 3 I (I):87-98.

38. Abraham C, Sheeran P, Johnston M: From health beliefs to selfregulation: Theoretical advances in the psychology of action control. Psychol Health 1998, 13:569-591.

39. Abraham C, Sheeran P, Norman P, Conner M, de Vries N, Otten W: When good intentions are not enough: Modeling postdecisional cognitive correlates of condom use. J Appl Soc Psychol 1999, 29(12):259|-26I2.

40. Bagozzi RP: The self-regulation of attitudes, intentions and behaviour. Soc Psychol Q 1992, 55: I 78-204.

4I. Hilberink SR, Jacobs JE, Schlösser M, Grol RP, de Vries H: Characteristics of patients with COPD in tree motivational stages related to smoking cessation. Patient Educ Couns 2006, 6I:449-457.

42. Locke EA, Latham GP: A theory of goal setting and task performance Englewood Cliffs, NJ: Prentice Hall; 1990.

43. Locke EA, Shaw KN, Saari LM, Latham GP: Goal-setting and task performance: 1969-1980. Psychol Bull 1981, 90:125-152. 
44. Bandura A, Simon KM: The role of proximal intentions in selfregulation of refractory behavior. Cognit Ther Res 1977, I: $177-193$.

45. Latham GP, Baldes J]: The "practical significance" of Locke's theory of goal setting. J Appl Psychol 1975, 60:122-124.

46. Brug J, Lechner $L$, de Vries $\mathrm{H}$ : Psychosocial determinants of fruit and vegetable consumption. Appetite 1995, 25:285-296.

47. de Nooijer J, de Vet E, Brug J, de Vries NK: Do implementation intentions help to turn good intentions into higher fruit intakes? J Nutr Educ Behav 2006, 38:25-29.

48. de Bruijn G-J, Kremers SP, de Vet E, de Nooijer J, van Mechelen W, Brug J: Does habit strength moderate the intention - behaviour relationship in the theory of planned behaviour? The case of fruit consumption. Psychol Health 2007, 22:899-916.

49. de Bruijn G-J, Kremers SP, Schaalma H, van Mechelen W, Brug J: Determinants of adolescent bicycle use for transportation and snacking behavior. Prev Med 2005, 40:658-667.

50. Cullen KW, Baranowski T, Smith SP: Using goal setting as a strategy for dietary behavior change. J Am Diet Assoc 200I, | 01:562-566.

5I. de Vries H, Kremers SP, Smeets T, Brug J, Eijmael K: The effectiveness of tailored feedback and action plans in an intervention addressing multiple health behaviors. Am J Health Promot 2008, 22:4I7-425.

52. Notwehr F, Snetselaar L, Yang J, Wu H: Stages of change for healthful eating and use of behavioral strategies. J Am Diet Assoc 2006, 106: 1035-104I.

53. Brink CL van den, Ocké MC, Houben AW, van Nierop P, Droomers M: Validering van standaardvraagstelling voeding voor Lokale en National Monitor Volksgezondheid (RIVM rapport 260854008). [Validation of a Community Health Services food consumption questionnaire in the Netherlands] Bilthoven: Rijksinstituut voor Volksgezondheid en Milieu (RIVM); 2005.

54. Martens MK, van Assema P, Brug J: Why do adolescents eat what they eat? Personal and social environmental predictors of fruit, snack and breakfast consumption among 12 - 14-yearold Dutch students. Public Health Nutr 2005, 8(8): I 258- 1265.

55. van Assema P, Brug J, Ronda G, Steenhuis I: The relative validity of a short Dutch questionnaire as a means to categorize adults and adolescents to total and saturated fat intake. J Hum Nutr Diet 200I, I 4:377-390.

56. Satorra A, Bentler PM: A scaled difference chi-square test statistic for moment structure analysis. Psychometrika 200I, 66(4):507-5।4.

57. Ferguson GA: Statistical analysis in psychology and education. Tokyo: McGraw-Hill; 1976.

58. Tabachnick BG, Fidell LS: Using multivariate statistics 4th edition. Boston, MA: Allyn and Bacon; 2001.

59. van Osch L, Reubsaet $A$, Lechner $L$, de Vries $H$ : The formation of specific action plans can enhance sun protection behavior in motivated parents. Prev Med 2008, 47: I27-132.

60. Liu LL, Park DC: Aging and medical adherence: the use of automatic processes to achieve effortful things. Psychol Aging 2004, 19(2):3|8-325.

61. Sheeran P, Orbell S: Implementation intentions and repeated behaviour: Augmenting the predictive validity of the theory of planned behavior. Eur J Soc Psychol 1999, 37:231-250.

62. Luszczynska A, Schwarzer R: Planning and self-efficacy in the adoption and maintenance of breast self-examination: A longitudinal study on self-regulatory cognitions. Psychol Health 2003, 18:93-108.

63. Orbell S, Sheeran P: Motivational and volitional processes in action initiation: A field study of the role of implementation intentions. J Appl Soc Psychol 2000, 30(4):780-797.

64. Wiedemann AU, Schüz B, Sniehotta FF, Scholz U, Schwarzer R: Disentangling the relation between intentions, planning, and behavior: A moderated mediation analysis. Psychol Health 2009, 24(I):67-79.

65. Kraemer H, Stice E, Kazdin A, Offord D, Kupfer D: How do risk factors work together? Mediators, moderators, and independent, overlapping and proxy risk factors. Am J Psychiatry 200I, I 58(6):848-856.

66. Kraemer H, Kiernan M, Essex M, Kupfer D: How and why criteria defining moderators and mediators differ between the Baron \& Kenny and MacArthur approaches. Health Psychol 2008, 27(2):SIOI-SI08.
67. MacKinnon DP: Introduction to statistical mediation analysis New York: Lawrence Erlbaum; 2008.

68. Fairchild AJ, MacKinnon DP: A general model for testing mediation and moderation effects. Prev Sci 2009, I 0(2):87-99.

69. van Osch L, Reubsaet A, Lechner L, Beenackers M, Candel M, de Vries $\mathrm{H}$ : Planning health behavior change: comparing the behavioral influence of two types of self-regulatory planning. $\mathrm{Br} J$ Health Psychol 2009 in press.

70. Sniehotta FF, Schwarzer R, Scholz U, Schüz B: Action planning and coping planning for long-term lifestyle change: Theory and assessment. Eur J Soc Psychol 2005, 35(4):565-576.

7I. Scholz U, Sniehotta FF, Burkert S, Schwarzer R: Increasing physical exercise levels: age-specific benefits of planning. J Aging Health 2007, I 9(5):85I-866.

72. Schüz B, Sniehotta FF, Wiedemann A, Seemann R: Adherence to a daily flossing regimen in university students: effects of planning when, where, how and what to do in the face of barriers. J Clin Periodontol 2006, 33(9):6|2-619.

73. van Osch L, Lechner L, Reubsaet A, Wigger S, de Vries H: Relapse prevention in a national smoking cessation contest: Effects of coping planning. Br J Health Psychol 2008, 13:525-535.

74. Cooper T, Cooke R, Schüz B, Sniehotta F: Motivational and volitional determinants of binge-drinking behaviour. Poster presented at the 2006 Student Members Groups Annual Conference of the British Psychological Society, Cardiff 2006.

75. Murgraff $V$, White $D$, Phillips $K$ : Moderating binge drinking: it is possible to change behaviour if you plan it in advance. Alcohol Alcohol 1996, 3 I (6):577-582.

76. Baranowski T, Weber Cullen K, Baranowksi J: Psychosocial correlates of dietary intake: Advancing dietary intervention. Annu Rev Nutr 1999, 19:17-40.

77. Brug J, de Vet E, de Nooijer J, Verplanken B: Predicting fruit consumption: Cognitions, intention, and habits. J Nutr Educ Behav 2006, 38:73-81.
Publish with Bio Med Central and every scientist can read your work free of charge

"BioMed Central will be the most significant development for disseminating the results of biomedical research in our lifetime. "

Sir Paul Nurse, Cancer Research UK

Your research papers will be:

- available free of charge to the entire biomedical community

- peer reviewed and published immediately upon acceptance

- cited in PubMed and archived on PubMed Central

- yours - you keep the copyright

Submit your manuscript here:

http://www.biomedcentral.com/info/publishing_adv.asp
BioMedcentral 